\title{
Porous Scaffolds Derived from Devitalized Tissue Engineered Cartilaginous Matrix Support Chondrogenesis of Adult Stem Cells
}

Henrique V. Almeida 1, 2, 3 , Anna D. Dikina ${ }^{4}$, Kevin J. Mulhall ${ }^{5}$, Fergal J. O'Brien ${ }^{1,6,7}$, Eben Alsberg 4, 8, 9 , Daniel J. Kelly 1, 2, 6, 7*

${ }^{1}$ Trinity Centre for Bioengineering, Trinity Biomedical Sciences Institute, Trinity College Dublin, 152-160 Pearse Street, Dublin 2, Ireland

2 Department of Mechanical and Manufacturing Engineering, School of Engineering, Trinity College Dublin, College Green, Dublin 2, Ireland

${ }^{3}$ Center for Neuroscience and Cell Biology, University of Coimbra, Rua Larga, 3004-504 Coimbra, Portugal

${ }^{4}$ Department of Biomedical Engineering, Case Western Reserve University, 10900 Euclid Ave. Cleveland, OH 44106, USA

${ }^{5}$ Sports Surgery Clinic, Northwood Avenue, Santry Demesne, Dublin 9, Ireland

${ }^{6}$ Tissue Engineering Research Group, Department of Anatomy, Royal College of Surgeons in Ireland, 123 St. Stephen's Green, Dublin 2, Ireland

7 Advanced Materials and Bioengineering Research Centre (AMBER), Trinity College Dublin \& Royal College of Surgeons in Ireland, Dublin 2, Ireland

${ }^{8}$ Department of Orthopedic Surgery, Case Western Reserve University, 10900 Euclid Ave. Cleveland, OH 44106, USA

9 The National Center for Regenerative Medicine, Division of Medical Sciences, Case Western Reserve University, 10900 Euclid Ave. Cleveland, OH 44106, USA

*Corresponding author: Daniel J. Kelly, Ph.D.

Address: Department of Mechanical and Manufacturing Engineering, School of Engineering, Parson's Building Trinity College Dublin, Dublin 2, Ireland

Telephone: +353-1-896-3947, Fax: +353-1-679-5554

E-mail address: kellyd9@tcd.ie 


\section{Abstract}

ECM-derived scaffolds have previously been developed from devitalized native cartilage and successfully used in tissue engineering. Such ECM based biomaterials are commonly derived from animal tissue, which may not represent the ideal source for applications in human. Native human ECM can be used as an alternative to xenogeneic tissue; however its supply may be limited leading to the need for a more readily available source of such biomaterials. The objective of this study was to compare devitalized native and tissue engineered cartilaginous ECM as chondro-permissive scaffolds for tissue engineering. To this end, porous scaffolds were produced using ECM derived from porcine articular cartilage and cartilaginous sheets engineered using human bone marrow stem cells. An identical process was used to produce scaffolds from three different types of devitalized ECMs, namely that derived from porcine cartilage (Native), from human engineered cartilaginous sheets (Eng) and from human engineered cartilaginous sheets generated in the presence of growth factor releasing microspheres (Eng-MS). Scaffolds produced using both devitalized engineered and native ECM possessed similar mechanical properties, pore size and GAG content, although were compositionally distinct. After being seeded with human infrapatellar fat pad stem cells, the engineered ECM derived scaffolds supported less robust cartilage matrix deposition than native ECM scaffolds. However, more chondro-permissive scaffolds could be generated using cartilaginous ECM engineered in the presence of TGF- $\beta 1$ releasing microspheres. These results demonstrate that engineered ECM can be used to produce scaffolds for cartilage tissue engineering, overcoming stock limitations and other barriers associated with native autogeneic, allogeneic and xenogeneic tissues. Such engineered ECM holds significant promise as an off-the-shelf chondro-permissive scaffold for articular cartilage repair.

Keywords: Tissue Engineering, Cartilage, ECM, Allogeneic, Scaffold, Stem Cells. 


\section{INTRODUCTION}

Articular cartilage is a complex avascular tissue with limited capacity for self repair ${ }^{1}$. Injuries to cartilage can be treated using different strategies, including cell-based therapies ${ }^{1 \mathrm{a}}$. Tissue engineering is a promising approach to regenerate tissues and organs through the use of cells, scaffolds, biochemical and biophysical cues ${ }^{2}$. Scaffolds can be engineered to facilitate cell adhesion, proliferation and consequent differentiation, often by mimicking aspects of the biochemical and biophysical characteristics of the native extracellular-matrix $(\mathrm{ECM})^{3}$. In particular, decellularized or devitalized ECM-derived scaffolds have been developed from numerous different tissues and used in regenerative medicine ${ }^{4}$. The success of ECM-derived scaffolds is dependent on the host response following implantation ${ }^{5}$. The immune response to such scaffolds is dependent on several factors, including fabrication methods and the origin of the ECM ${ }^{5}$.

Cartilage ECM-derived scaffolds have been shown to be chondro-permissive and to facilitate articular cartilage repair ${ }^{4 a}$.. In general, however, such approaches rely on the use of animal-derived tissues (xenogeneic), with the associated risk of an adverse immune response and disease transmission after implantation into human ${ }^{7}$. As an alternative to the use of xenogeneic tissues, native human cartilage ECM (either autogeneic or allogeneic) has also been used as a scaffold for joint regeneration ${ }^{7 a, 8}$. However, the supply of such native human tissues is limited, and there are concerns related to disease transmission with allogeneic approaches, motivating the use of in vitro engineered cartilage ECM from human chondrocytes or mesenchymal stem cells (MSCs) ${ }^{9}$. In addition to lowering the risk of disease transmission and eliciting an adverse immune response, engineered ECM-derived scaffolds could be considered to be more developmentally immature and hence provide a milieu of features more conductive to chondrogenesis ${ }^{9 \mathrm{~b}}$. Engineered cartilage ECM is compositionally different to native articular cartilage, which impacts how cells and growth factors interact 
with scaffolds generated using such biomaterials ${ }^{3 \mathrm{~d}}$. For example, chordin-cysteine binding sites, which are not available in mature cartilage ECM, can bind to transforming growth factor-beta (TGF- $\beta$ ) family growth factors in more immature tissue ${ }^{3 \mathrm{~d}}$. This interaction between collagen and TGF- $\beta$ is expected to regulate chondrogenesis of stem cells 9 , 10 .

The goal of this set of experiments was to compare devitalized native and engineered cartilage ECM as chondro-permissive scaffolds for cartilage tissue engineering. Engineered tissues were produced from high density cultures of bone marrow MSCs that self-assembled to generate cartilaginous constructs ${ }^{11}$. To enhance chondrogenesis, TGF- $\beta 1$ releasing gelatin microspheres were combined with these MSCs ${ }^{9 c}$. These microspheres, when included in high density sheets of MSCs, can uniformly deliver chondrogenic growth factor over a sustained period, enabling homogenous differentiation and robust cartilage tissue formation compared to supplementing the media with growth factor ${ }^{9 \mathrm{c}}$. Therefore the goal for this study was to evaluate if scaffolds produced using ECM engineered in the presence of such TGF- $\beta 1$ loaded microspheres would be more chondro-permissive. Hence, we sought to develop and assess the chondro-inductivity of ECM-derived scaffolds generated from three different types of ECM: (1) porcine ECM (Native), (2) in vitro engineered sheets of cartilage (Eng) and (3) in vitro engineered cartilage sheets generated in the presence of growth factor releasing microspheres (Eng-MS), see Figure 1. To access the potential of these different scaffolds to support chondrogenesis, infrapatellar fat pad human stem cells (FPSCs) were incorporated onto the ECM matrices and chondrogenesis assessed over 28 days. An additional goal of the current set of experiments was to assess the potential of engineered ECM scaffolds to perform as growth factor delivery platform to enhance chondrogenesis of adult stem cells. 


\section{MATERIAL AND METHODS}

\subsection{Harvest of Porcine Cartilage ECM}

Native cartilage tissue was harvested in aseptic conditions as previously described ${ }^{6 \mathrm{~b} \text {, }}$ ${ }^{6 c}$. Briefly, porcine articular cartilage was collected in femoral condyles, the patella groove and the tibial plateau of pigs (Female; 3 month) using a biopsy punch (diameter $8 \mathrm{~mm}$ ). The pigs were not sacrificed specifically for this study; the cartilaginous tissue was harvested from the pigs after they were sacrificed for an unrelated study. Cartilage was washed and kept in phosphate buffered saline (PBS; Sigma-Aldrich) containing penicillin (100 $\left.\mathrm{U} \mathrm{ml}^{-1}\right)$ streptomycin $\left(100 \mu \mathrm{g} \mathrm{ml}^{-1}\right.$; GIBCO, Biosciences $)$.

\subsection{Engineering of Human Cartilaginous ECM using Bone Marrow-derived Mesenchymal Stem Cells}

As previously described ${ }^{12}$, human MSCs were provided by Case Comprehensive Cancer Center Hematopoietic Biorepository and Cellular Therapy Core under University Hospitals of Cleveland Institutional Review Board approval. Firstly, bone marrow aspirates were washed (with Dulbecco's Modified Eagle Medium Low Glucose (DMEM-LG; SigmaAldrich, St. Louis, MO) with 10\% bovine serum (Gibco Qualified FBS; Life Technologies, Carlsbad, CA) and $100 \mathrm{U} \mathrm{ml}^{-1}$ penicillin-streptomycin (Corning Inc, Corning, NY)). By using a Percoll gradient (Sigma-Aldrich), mononuclear cells were separated, plated and cultured in an incubator $\left(37^{\circ} \mathrm{C} ; 5 \% \mathrm{CO}_{2}\right)$. Cells were supplemented with expansion media with $10 \mathrm{ng} \mathrm{ml}^{-}$

${ }^{1}$ fibroblast growth factor-2 (FGF-2; R\&D Systems, Minneapolis, MN) twice a week. MSCs were cultured to passage 3 , and used in this study to engineer human cartilage sheets ${ }^{9 c}$. Transwell inserts $(12 \mathrm{~mm}$; Corning) were incubated with $0.75 \mathrm{ml}$ expansion media in each well $\left(2\right.$ hours; $\left.37^{\circ} \mathrm{C}\right)$ after which an additional $0.75 \mathrm{ml}$ of chondrogenic pellet media (CPM) 
with Dulbecco's Modified Eagle Medium High Glucose (DMEM-HG; Sigma-Aldrich), 1\% ITS+ Premix (Corning Inc), $10^{-7} \mathrm{M}$ dexamethasone (MP Biomedicals, Solon, OH), $1 \mathrm{mM}$ sodium pyruvate (HyClone Laboratories), $100 \mathrm{mM}$ non-essential amino acids (Lonza Group, Switzerland), $37.5 \mathrm{mg} \mathrm{ml}^{-1}$ ascorbic acid-2-phosphate (Wako Chemicals USA), $100 \mathrm{U} \mathrm{ml}^{-1}$ penicillin-streptomycin (Corning) and $10 \mathrm{ng} \mathrm{ml}^{-1}$ TGF- $\beta 1$ (Peprotech, Rocky Hill, NJ) was added to the plate well. Next, 2 million hMSCs were suspended in $500 \mu$ of CPM and cultured in transwell inserts in an incubator $\left(48 \mathrm{~h} ; 37^{\circ} \mathrm{C} ; 5 \% \mathrm{CO}_{2}\right)$. TGF- $\beta 1$ was supplemented to the culture medium. Following $24 \mathrm{~h}$, medium of each well was replaced by fresh $1.5 \mathrm{ml}$ CPM. A complete media change $(2 \mathrm{ml})$ occurred after 48 hours and every 2 days thereafter, for a culture period of 4 weeks ${ }^{9 c}$.

Microspheres (Gelatin, 11.1 w/v\% Type A; Sigma-Aldrich) were produced in a waterin-oil emulsion ${ }^{9 c, 12}$. Microspheres were crosslinked with genipin (2 hours; $1 \mathrm{w} / \mathrm{v} \%$; Wako Chemicals, USA), washed with deionised water, lyophilized and rehydrated with PBS (HyClone Laboratories, Logan, UT) with TGF- $\beta 1$ (400 ng mg-1 of microspheres; 2 hours, $\left.37^{\circ} \mathrm{C}\right)^{9 \mathrm{c}}$. High density MSC sheets containing microspheres were assembled in the same manner as the normal human engineered sheets mentioned earlier in the text, except without TGF- $\beta 1$ supplementation in the media. Specifically, microspheres $(1.5 \mathrm{mg})$ and MSCs (2 million) were suspended in $500 \mu$ of basal pellet medium (BPM; CPM without TGF- $\beta 1$ ) and seeded onto membranes of transwell inserts. Culture conditions were the same as the human engineered sheets, however with no TGF- $\beta 1$ supplementation.

\subsection{Preparation of Cartilage ECM-derived Scaffolds}

Cartilage-derived ECM scaffolds were fabricated in aseptic conditions as previously reported ${ }^{6 \mathrm{~b}}$. First, cartilage (native and engineered) was cut with a sharp blade, each group separately. Cartilage slurry was created by pulverizing cartilage using a cryogenic mill (6770 
Freezer/Mill, SPEX, UK). Pulverized cartilage was then homogenized in UPW by a homogenizer (IKAT10, IKA Works, USA) to generate a fine slurry. Scaffolds were fabricated using a previously used know slurry concentration $\left(250 \mathrm{mg} \mathrm{ml}^{-1}\right)^{6 \mathrm{~b}}$. Cartilage slurry was poured into moulds $(60 \mu \mathrm{l})$ and lyophilized (FreeZone Triad, Labconco) ${ }^{6 \mathrm{~b}, 6 \mathrm{c}}$. The scaffolds were crosslinked by dehydrothermal (DHT) and 1-Ethyl-3-3-dimethyl aminopropyl carbodiimide (EDAC) ${ }^{6 b}, 13$. DHT method was carried out in an oven (VD23, Binder, Germany), at $115^{\circ} \mathrm{C}$ and 2 mbar (24 hours). EDAC (Sigma-Aldrich) crosslinking was performed (2 hours; $6 \mathrm{mM}$ ) with N-Hydroxysuccinimide (NHS; Sigma-Aldrich). It was used a molar ratio of 2.5 M EDAC/M N-Hydroxysuccinimide ${ }^{13 \mathrm{~b}}$. Identical process of fabrication of the scaffolds was performed for the three different types of ECM: derived from porcine ECM (Native), from human engineered sheets (Eng) and from human engineered sheets with microspheres (Eng-MS) (Figure 1). 


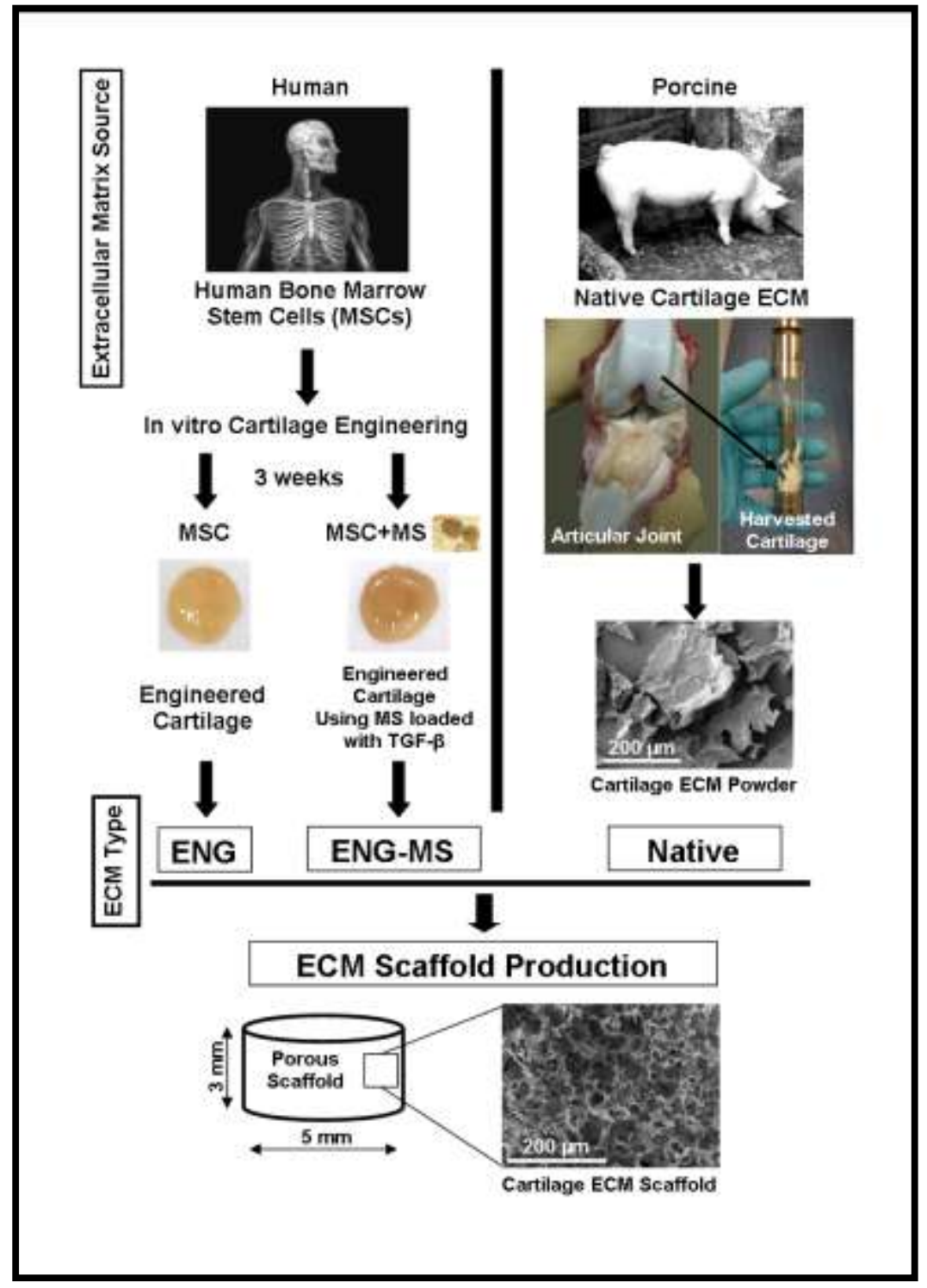

Figure 1. Schematic of cartilage ECM origin and scaffold production; MS: microspheres; FPSCs: human infrapatellar fat pad-derived stem cells.

\subsection{Scanning Electron Microscopy, Mean Pore Size and Mechanical Testing}

To analyze such porous scaffolds, scanning electron microscopy (SEM) was used. Briefly, structures were fixed in $4 \%$ paraformaldehyde solution (PFA, Sigma-Aldrich) overnight. Secondly, scaffolds were dehydrated (ethanol 10-100\%), and examined under a scanning electron microscope (SEM; Tescan Mira FEG-SEM XMU, Libušina, Czech Republic). Pore size quantification was performed by calculating the diameter of numerous pores (40; in Image J) in SEM micrographs of the scaffolds $(n=3)$ previous to cell seeding ${ }^{6 b}$, 
14. Scaffolds (dry and acellular; $5 \mathrm{~mm}$ diameter; $3 \mathrm{~mm}$ height) were assessed in terms of mechanical performance with a $5 \mathrm{~N}$ load cell (Zwick Z005, Roell, Germany) ${ }^{15}$. Specifically, assessment was preceded by a $0.03 \mathrm{~N}$ load to allow proper contact with the loading platens. Additionally, samples were subjected to compressive strain $(10 \% ; 0.001 \mathrm{~mm} / \mathrm{s})$, and the Young's modulus was determined (slope of the stress-strain curve). After a four week culture period cartilaginous constructs were assessed mechanically (compressive strain, 10\%; 0.001 $\mathrm{mm} / \mathrm{s}$ ) in a bath of PBS (RT) until equilibrium was reached, as previously described ${ }^{15}$.

\subsection{Cell Isolation and Culture}

The consent for the use of human infrapatellar fat pad (FP) stromal cells was obtained from the ethical review board of the Mater Misericordiae Hospital (Ref: 1/378/1501). Cells were obtained from a biopsy of a FP, from a ligament reconstruction surgery from a patient without osteoarthritis. The FP tissue was weighed and washed in PBS, as previously described ${ }^{6 \mathrm{~b}}$. Tissue was diced and rotated at $37^{\circ} \mathrm{C}$ in DMEM-HG GlutaMAXTM (GIBCO, Biosciences, Ireland) with type II collagenase (750 $\mathrm{U} \mathrm{ml}^{-1}$, Worthington Biochemical, LaganBach Services) and $1 \%$ penicillin $\left(100 \mathrm{U} \mathrm{ml}^{-1}\right)$-streptomycin $\left(100 \mu \mathrm{g} \mathrm{ml}^{-1}\right)$ for four hours. A ratio of $4 \mathrm{ml} / \mathrm{g}$ of tissue of collagenase was found to be optimal ${ }^{16}$. Cells were washed, passed through a cell strainer $(40 \mu \mathrm{m})$ and centrifuged (650 g; 5 minutes). The remaining cells were plated (5000 cells $\mathrm{cm}^{-2}$ ). The cell population was cultured (to P2) in DMEM-HG with 10\% FBS and 1\% penicillin $\left(100 \mathrm{U} \mathrm{ml}^{-1}\right)$-streptomycin $\left(100 \mathrm{mg} \mathrm{ml}^{-1}\right)$ with FGF-2 (5 ng ml ${ }^{-1}$; ProSpec-Tany TechnoGene Ltd, Israel).

$0.5 \times 10^{6}$ human FPSCs were seeded into scaffolds $(\mathrm{d}=5 \mathrm{~mm} ; \mathrm{h}=3 \mathrm{~mm})$. Constructs were in chondrogenic medium ${ }^{6 b, 6 c, 16 b}$, for four weeks $\left(5 \% \mathrm{O}_{2} ; 37^{\circ} \mathrm{C}\right)$, kept in 12 -well plates, and each was placed within agarose moulds $(3 \% \mathrm{w} / \mathrm{v}$; Sigma-Aldrich, Ireland; $\mathrm{d}=5 \mathrm{~mm})$ to stop cell movement to the wells and increase cell-seeding efficiency. Chondrogenic medium 
consisted of DMEM GlutaMAXTM with penicillin $\left(100 \mathrm{U} \mathrm{ml}^{-1}\right)$-streptomycin $\left(100 \mu \mathrm{g} \mathrm{ml}^{-1}\right)$, sodium pyruvate $\left(100 \mu \mathrm{g} \mathrm{ml}^{-1}\right)$, L-proline $\left(40 \mu \mathrm{g} \mathrm{ml}^{-1}\right)$, L-ascorbic acid-2-phosphate $(50 \mu \mathrm{g}$ $\left.\mathrm{ml}^{-1}\right)$, bovine serum albumin $\left(1.5 \mathrm{mg} \mathrm{ml}^{-1}\right)$, insulin-transferrin-selenium $(1 \mathrm{x})$, dexamethasone $(100 \mathrm{nM})$ (all from Sigma-Aldrich, Ireland) and transforming growth factor- $\beta 3$ (TGF- $\beta 3,10$ ng $\mathrm{ml}^{-1}$; ProSpec-Tany TechnoGene Ltd, Israel).

In addition to adding TGF- $\beta 3$ into the media, we also examined if this growth factor could instead be soak loaded onto the scaffold. In this way, the ECM construct would deliver TGF- $\beta 3$. For these groups (Eng+ and Eng-MS+) no TGF- $\beta 3$ was additionally supplemented during culture. Instead, TGF- $\beta 3$ (200 ng), in $40 \mu 1$ of medium, was soak-loaded into the ECM-derived scaffold with cells for 15 minutes. After seeding the scaffold with cells were left in the incubator for 2 hours. Furthermore, $2.5 \mathrm{ml}$ of chondrogenic media (without TGF$\beta 3)$ was added, and repeated twice a week (2.5 $\mathrm{ml}$ per well).

\subsection{Biochemical analysis}

Biochemical analyses were performed for day 0 and 28 constructs, for glycosaminoglycan (sGAG), collagen, and DNA content, as presented elsewhere ${ }^{6 c, 14}$. Constructs were digested by incubation in papain $\left(125 \mu \mathrm{g} \mathrm{ml}^{-1}\right)$ in sodium acetate $(0.1 \mathrm{M})$, cysteine-HCl (5 mM), Ethylenediaminetetraacetic acid (EDTA) (0.05 M), pH 6.0 (all from Sigma-Aldrich, Ireland) at $60^{\circ} \mathrm{C}(10 \mathrm{rpm} ; 18$ hours $)$. DNA content of each sample was determined with Hoechst Bisbenzimide 33258, with a calf thymus DNA standard ${ }^{14}$. Proteoglycan content was determined by sGAG quantification in constructs with dimethylmethylene blue dye-binding assay (Blyscan, Biocolor Ltd.). Collagen was quantified by hydroxyproline content, following hydrolysis $\left(110^{\circ} \mathrm{C} ; 18\right.$ hours) in $\mathrm{HCL}(38 \%)$, and assayed using chloramine-T assay with a hydroxyproline/collagen ratio of 1:7.69 ${ }^{16 a}$. All 
experimental data presented corresponds to a minimum of four analyzed constructs per group, and all the experiments were repeated twice.

\subsection{Histology and Immunohistochemistry}

As previously described ${ }^{6 \mathrm{~b}, 6 \mathrm{c}}$, constructs were fixed $\left(4^{\circ} \mathrm{C}\right)$ in paraformaldehyde $(4 \%)$ (Sigma-Aldrich), followed by washing steps and paraffin embedded. Samples were sectioned and dyed with picro-sirius red for collagen, 1\% alcian blue 8GX (Sigma-Aldrich) in $\mathrm{HCl}(0.1$ M) for sGAG , and with $0.1 \%$ nuclear fast red solution (Sigma-Aldrich) for cell nuclei. To image the orientation/organization of the collagen fibril, picro-sirius red dyed samples were analyzed with in polarized light (Nikon Eclipse E400 POL), as previously described ${ }^{17}$. Alizarin calcium staining was performed to assess calcium accumulation ${ }^{18}$. As previously described $^{6 \mathrm{c}, 16 \mathrm{~b}}$, immunohistochemical assessment was performed with monoclonal antibody for type I, II and X collagen (Abcam, UK). After PBS wash, sections were subjected to peroxidase activity (20 minutes), and with chondroitinase $\mathrm{ABC}\left(1\right.$ hour, $37^{\circ} \mathrm{C}$; Sigma, $0.25 \mathrm{U}$ $\mathrm{ml}^{-1}$ ). Slides were blocked with $10 \%$ goat serum (30 minutes) and incubated with mouse monoclonal anti-collagen type I, II and X (1 $\mathrm{mg} \mathrm{ml}^{-1}$; 1 hour at RT; Abcam, UK). A secondary antibody binding was then applied $\left(1 \mathrm{mg} \mathrm{ml}^{-1}\right.$; 1 hour; Anti-Mouse IgG Biotin antibody produced in goat). Vectastain $\mathrm{ABC}$ reagent (Vectastain $\mathrm{ABC}$ kit, Vector Laboratories, UK) for 5 minutes in peroxidase DAB substrate kit (Vector laboratories, UK) allowed color alteration. Histological sections were mounted by using Vectamount medium (Vector Laboratories, UK).

\subsection{Measurement of TGF- $\beta 3$ present in ECM scaffolds}

The TGF- $\beta 3$ present in the ECM-derived scaffolds before seeding with FPSC was measured using a previously reported protocol ${ }^{19}$. Briefly, the scaffolds were treated with a 
solution of $4 \mathrm{M}$ guanidine hydrochloride (Pierce) at $4^{\circ} \mathrm{C}$ for 2 days to extract any growth factors. TGF- $\beta 3$ content was determined by ELISA ${ }^{6 c, 19-20}$. By following manufacturer instructions, capture antibody $\left(360 \mu \mathrm{g} \mathrm{ml}^{-1}\right)$ was in 96 well plates (mouse anti-human TGF$\beta 3$; R\&D Systems, UK). The samples and standards (ProSpec-Tany TechnoGene Ltd, Israel) were incubated (2h). Furthermore, detection antibody $\left(18 \mu \mathrm{g} \mathrm{ml}^{-1}\right.$ of biotinylated goat antihuman TGF- $\beta 3$ ) was added and incubated (2h). Next, the plate was washed, dried and incubated in streptavidin-HRP (horseradish-peroxidase; R\&D Systems, UK). Substrate solution (1:1 mixture of $\mathrm{H}_{2} \mathrm{O}_{2}$ and tetramethylbenzidine; R\&D Systems, UK) was added to each well, followed by incubation (no light). Stop solution $\left(2 \mathrm{~N} \mathrm{H}_{2} \mathrm{SO}_{4}\right.$; Sigma-Aldrich) was added and the optical density was determined (450 $\mathrm{nm})$.

\subsection{Statistical Analysis}

Statistical results were obtained with MINITAB 15.1 software package (Minitab Ltd., Coventry, UK), and presented as mean \pm SD. Groups were analyzed for significant differences using a general linear model for analysis of variance (ANOVA) with factors including ECM and growth factor delivery. Tukey's test for multiple comparisons was used, with significance accepted for $\mathrm{p}<0.05$.

\section{RESULTS}

\subsection{Engineered and native cartilage ECM are compositionally distinct}

Porous scaffolds were fabricated using three different cartilage ECM derived materials: (1) native porcine articular cartilage (Native); (2) engineered cartilage (Eng); and (3) cartilage engineered with TGF- $\beta 1$ loaded microspheres (Eng-MS). Prior to forming scaffolds using these materials, their ECM composition was compared (Figure 2). Both the 
Eng and Eng-MS tissues stained strongly for glycosaminoglycans (Figure $2 \mathrm{G}$ and L), comparable to native articular cartilage (Figure 2B). Native tissue stained more intensely for collagen compared to engineered tissues (Figure 2C, H, and M). Polarized light microscopy (PLM) revealed that engineered tissues did not possess the same degree of cartilage fibril organization as native articular cartilage (Figure 2D). All tissues stained negatively for calcium accumulation (Figure $2 \mathrm{E}, \mathrm{J}$ and $\mathrm{O}$ ).

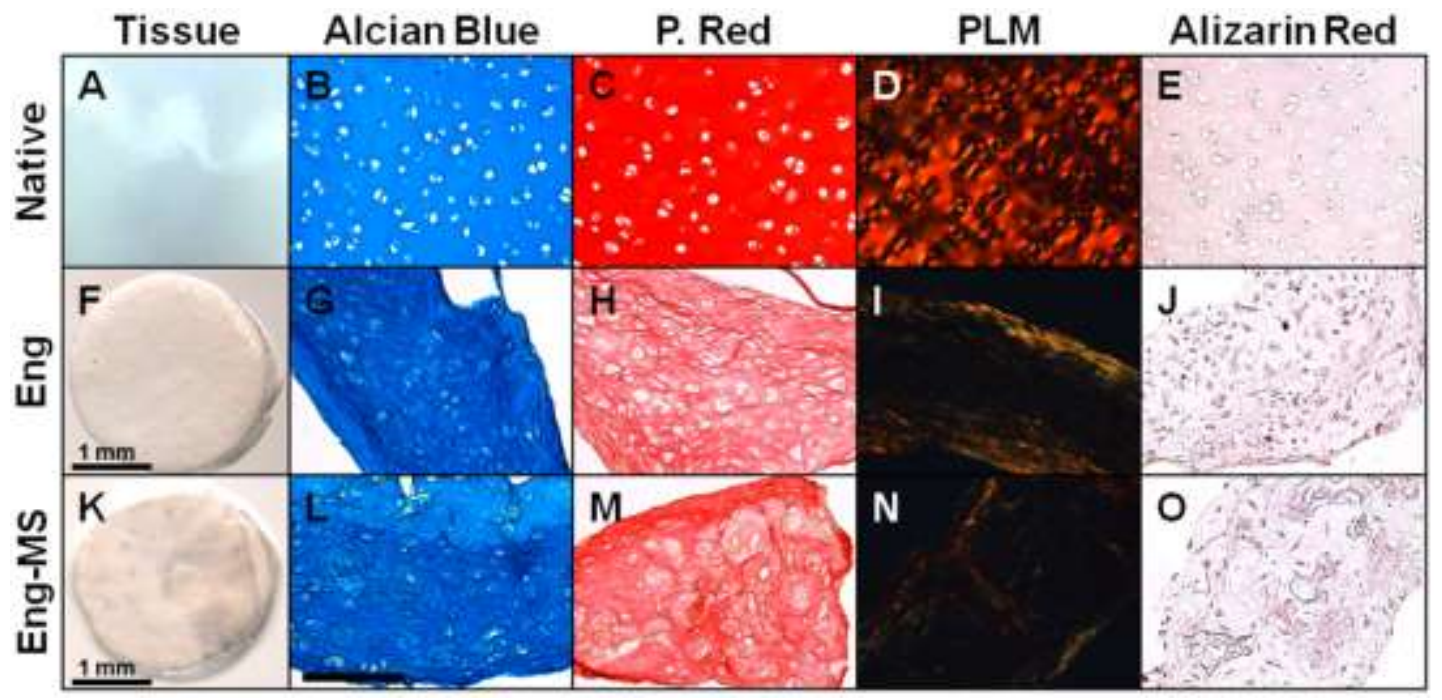

Figure 2. Macroscopic appearance of native (Native - A), engineered (Eng - F) and engineered with microspheres (Eng MS - K) cartilage. Histological staining for alcian blue and picro-sirius red (P. Red) for native (B, C), Eng (G, H) and Eng-MS (L, M) cartilage groups. Polarized light microscopy (PLM) micrographs of collagen fibrils architecture for native (D), Eng (I) and Eng-MS (N) cartilage. Alizarin red calcium staining for native (E), Eng (J) and Eng-MS (O) cartilaginous tissues. (A, F, K) Scale bar: $1 \mathrm{~mm}$; (B-E, G-J, L-O) Scale bar histology: $50 \mu \mathrm{m}$.

Immunohistochemistry was used to determine the specific types of collagen within the different ECMs. The native, Eng and Eng-MS tissues all stained weakly for type I collagen deposition (Figure 3A, D, and G). As expected, native cartilage stained strongly for type II collagen (Figure 3B), but engineered ECM stained less intensely. Eng-MS constructs stained slightly more intensely for type II collagen than the Eng group (Figure 3H). Weak staining for type $\mathrm{X}$ collagen was present in native and Eng (Figure 3C and F) tissues. Type $\mathrm{X}$ collagen staining was slightly more intense in the Eng-MS group (Figure 3I). 


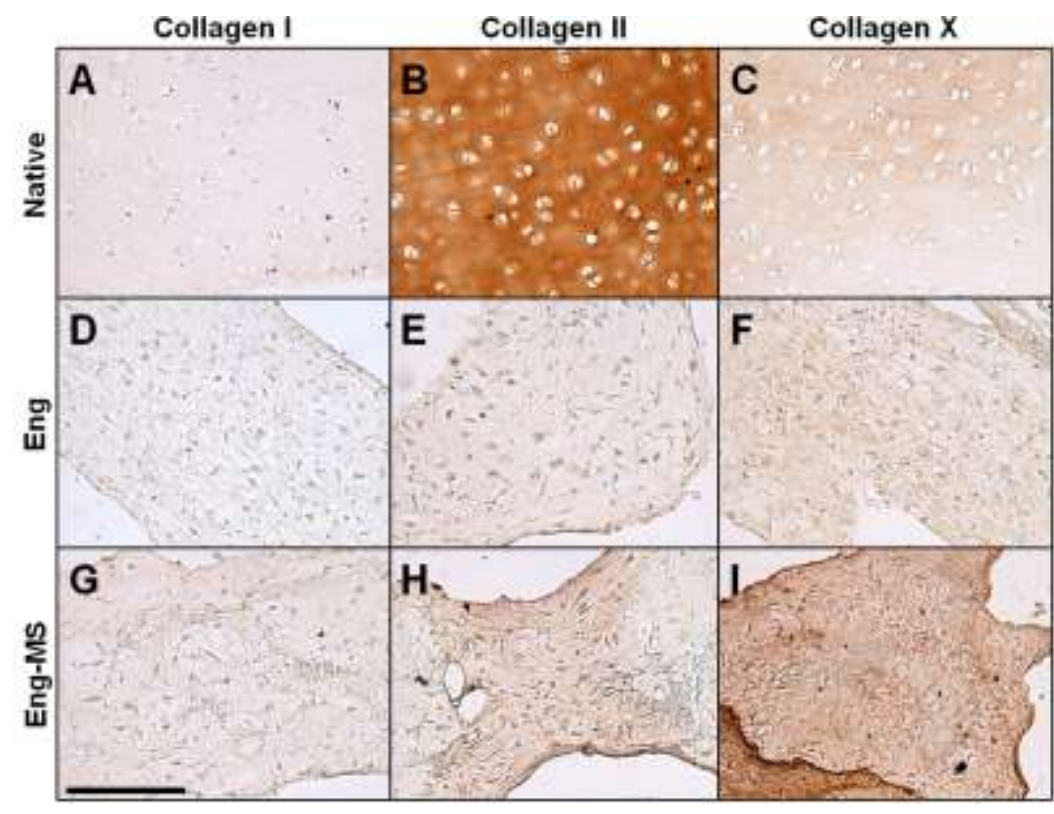

Figure 3. Immunohistochemical analysis for type I, II and X collagen for native (A-C), Eng (D-F), and Eng-MS (G-I). Scale bar: $50 \mu \mathrm{m}$.

\subsection{Native and engineered (Eng and Eng-MS) ECM-derived scaffolds support chondrogenesis of stem cells}

Porous scaffolds were firstly produced by a previously described freeze drying protocol $^{6 \mathrm{~b}}$ using devitalized native (Native) and engineered ECM (Eng and Eng-MS). There were no significant differences in collagen and GAG levels and morphology between Eng and Eng-MS scaffolds (data not shown), so only data for Native and Eng scaffolds are presented here. SEM was used to characterize the porosity of both native and Eng scaffolds (Figure 4A-D). The architecture (Figure 4A-D), porosity, Young's modulus (Native: $34 \pm 8$ $\mathrm{kPa}$; Eng: $30 \pm 4 \mathrm{kPa}$; Figure $4 \mathrm{E})$ and mean pore size $(\sim 70 \mu \mathrm{m})$ was comparable for all scaffold types (Figure 4F). Both native and Eng scaffolds stained positive for GAG (Figure 4G and I), collagen (Figure $4 \mathrm{H}$ and $\mathrm{J}$ ), with the native scaffolds staining more intensely for type II collagen than Eng group (Figure 4K and L). The GAG content was slightly higher for the Eng scaffolds (Native: $46 \pm 11 \mu \mathrm{g}$ and Eng: $66 \pm 17 \mu \mathrm{g}$ ), while the collagen content of the 
native ECM-derived scaffolds was higher than the Eng group (Native: $335 \pm 69 \mu \mathrm{g}$ and Eng: $104 \pm 80 \mu \mathrm{g})$.
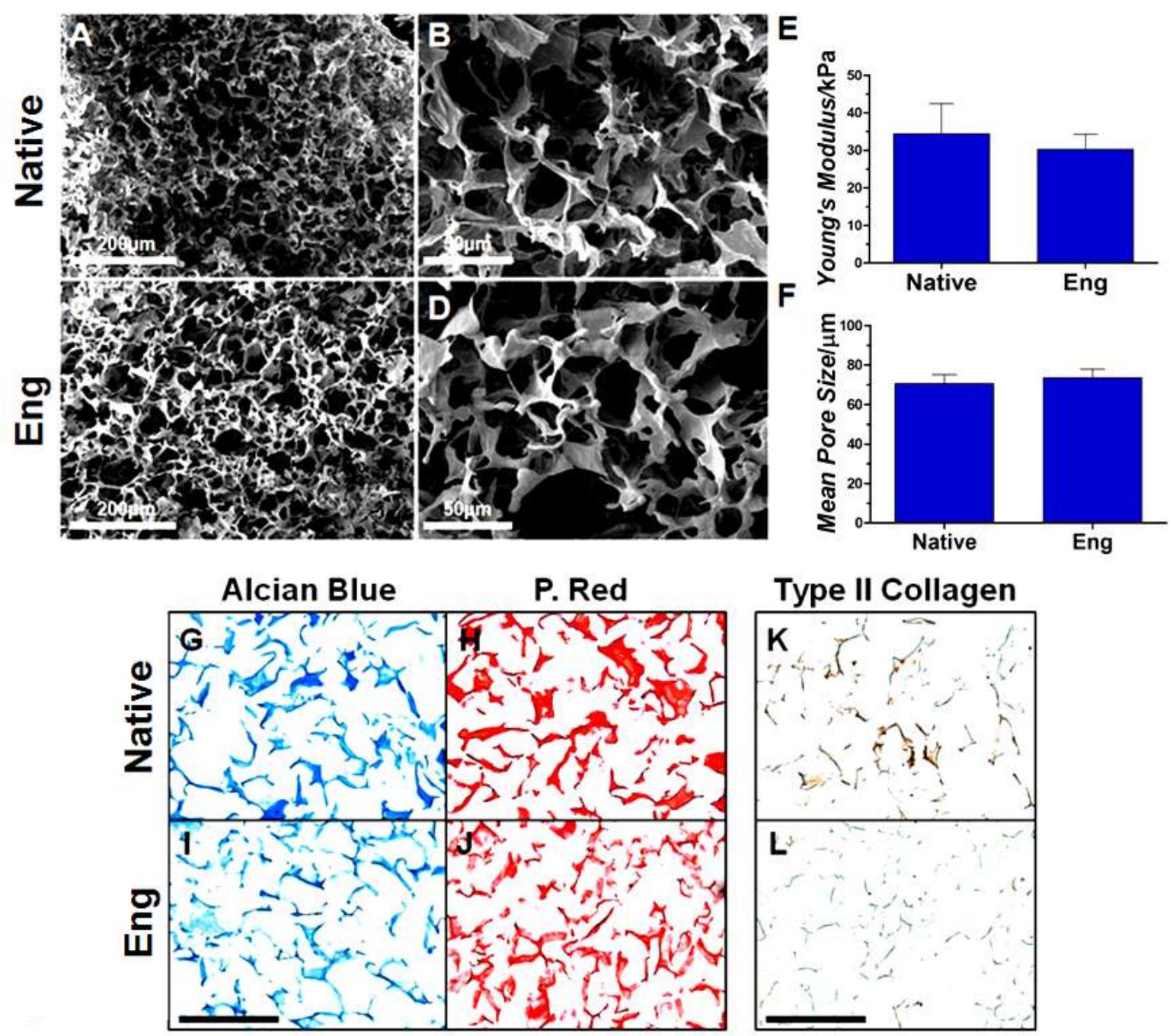

Figure 4. Scanning electron microscopy (SEM) micrographs for native (A, B) and Eng (C, D) ECMderived scaffolds. Young's modulus (E) and mean pore size (F) for both native and engineered groups $(n=4)$. Acellular ECM-derived scaffolds histological staining for alcian blue, picro-sirius red (P. Red), and type II collagen for native (G, H, K) and Eng (I, J, L) groups. All micrographs are for the dry scaffolds before culture period. (G-L) Scale bar: $50 \mu \mathrm{m}$.

TGF- $\beta 3$ quantification was performed using ELISA to quantify the amount of growth factor present in each of the scaffold groups. Higher levels of TGF- $\beta 3$ were present in 
scaffolds engineered using Eng-MS ECM compared with the other groups, suggesting that incorporation of TGF- $\beta 1$ releasing microspheres enhanced TGF- $\beta 3$ production and accumulation within the engineered tissue and/or retention of the growth factor within the matrix throughout scaffold fabrication (Figure 5M).

To assess the capacity of the scaffolds to support chondrogenesis, they were seeded with FPSCs and maintained in culture for 4 weeks in media continuously supplemented with TGF- $\beta 3$. In such conditions, both native and engineered (Eng and Eng-MS) scaffolds supported the development of engineered tissues that resembled cartilage macroscopically (Figure 5A, E and I). Histological analysis suggested that GAG and collagen deposition was higher within native ECM scaffolds compared to the engineered ECM scaffolds (Figure 5B and C). In addition, type II collagen deposition was more intense in native cartilage ECM scaffolds (Figure 5D). When comparing the engineered ECM scaffold groups, type II collagen immuno-staining appeared slightly more intense in tissues engineered within the Eng-MS ECM-derived scaffolds (Figure 5L). Quantitative biochemical assays revealed that the DNA, GAG and collagen content was higher in the native scaffolds when compared to the Eng group $(* * * p<0.001$; Figure $5 \mathrm{~N}-\mathrm{P})$, although no differences in GAG accumulation was observed between the native and Eng-MS scaffolds $(* * * p<0.001$; Figure $5 \mathrm{~N}-\mathrm{P})$. In addition, the Eng-MS ECM-derived constructs accumulated higher levels of GAG and collagen compared to the Eng scaffolds (Figure 5O). The equilibrium moduli of the three different constructs were not statistically different (Figure 5Q). Finally, alizarin red staining revealed no calcification in any of the engineered groups (data not shown). 


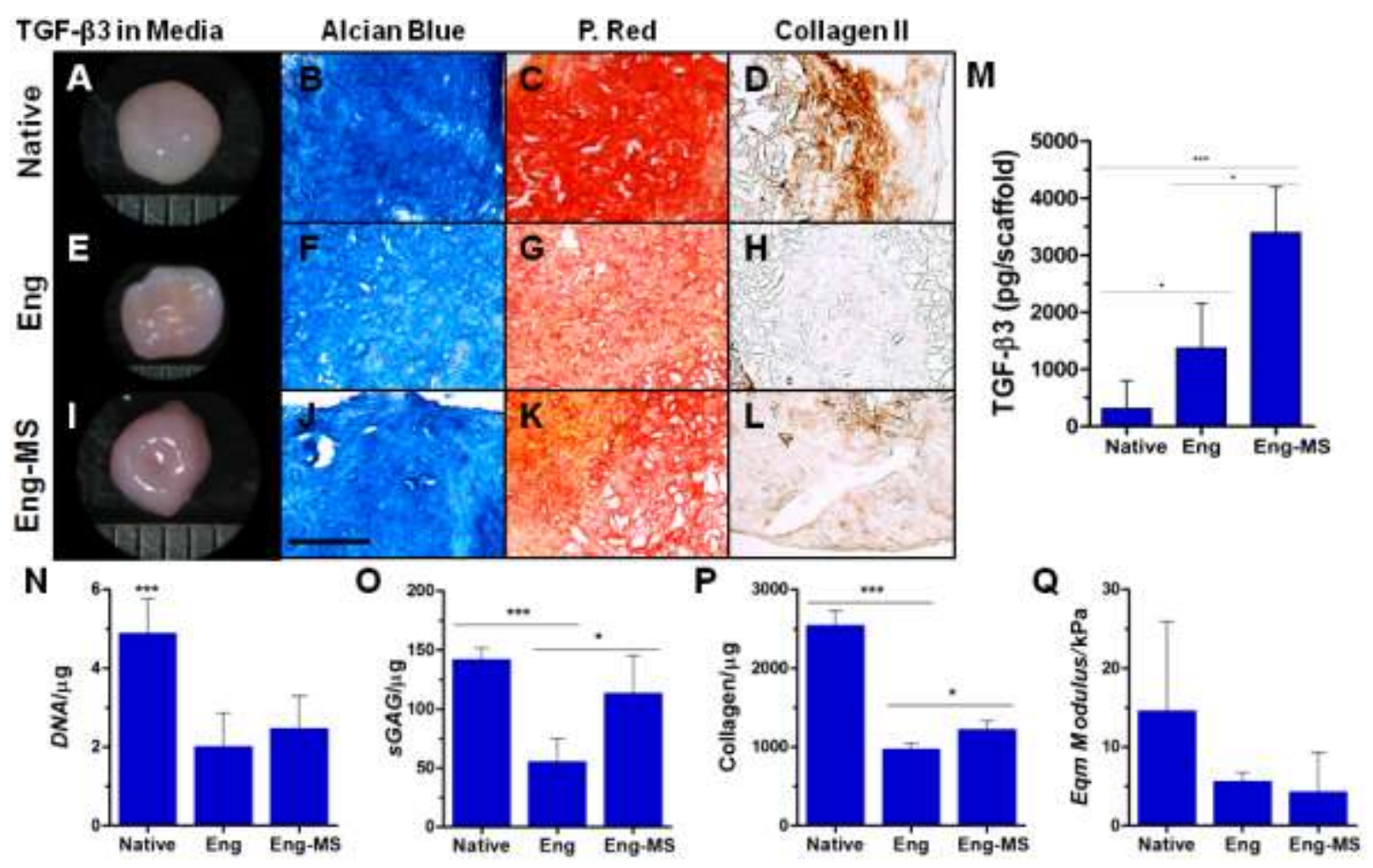

Figure 5. Macroscopic appearance of native (A), Eng (E) and Eng-MS (I) ECM-derived constructs after 28 days in culture (TGF- $\beta 3$ in media). Histological staining for alcian blue, picro-sirius red (P. Red) and type II collagen for native (B-D), Eng (F-H) and Eng-MS (J-L) groups. All micrographs are for 28 days culture period. TGF- $\beta 3$ content (ELISA) (M) of the native, Eng and Eng-MS ECMderived scaffolds before culture period $\left(\mathrm{n}=4 ;{ }^{*} \mathrm{p}<0.05 ; * * * \mathrm{p}<0.001\right)$. DNA (N) (day 0 scaffolds values were subtracted from the total DNA content: Native- $1.9 \pm 0.6 \mu \mathrm{g}$, Eng- $2.9 \pm 0.4 \mu \mathrm{g}$ and Eng-MS 2.5 $\pm 0.2 \mu \mathrm{g})$, GAG (O), Collagen (P) and Equilibrium Modulus (Q) for native, Eng and Eng-MS groups for 28 days culture period $\left(\mathrm{n}=5 ;{ }^{*} \mathrm{p}<0.05 ; * * * \mathrm{p}<0.001\right)$. Scale bar: $50 \mu \mathrm{m}$.

\subsection{Engineered ECM derived scaffolds can be used as TGF- $\beta 3$ releasing systems to} promote chondrogenesis of adult stem cells

It was shown that native cartilage ECM scaffolds can act as reservoirs of TGF- $\beta 3$, inducing robust chondrogenesis of FPSCs ${ }^{6 b, 6 c}$. To assess if engineered cartilage ECM scaffolds could perform in a similar manner, these were laden with growth factor (TGF- $\beta 3$ ), seeded with FPSCs and maintained in culture for 28 days. No other exogenous growth factor 
was added to the medium for the rest of the culture period. At the end of this 28 day culture period, the Eng+ and Eng-MS+ constructs resembled cartilage macroscopically (Figure 6A and E). GAG and collagen histological staining was similar for the Eng+ and Eng-MS+ groups (Figure 6B, C, F, and G). Immunohistochemistry results for type II collagen was similar for constructs fabricated using Eng+ and Eng-MS+ engineered cartilage (Figure 6D and $\mathrm{H})$. Biochemical sGAG quantification was comparable for Eng+ and Eng-MS+ constructs (Figure 6I), and of a similar order of magnitude to that observed when growth factor was continuously supplemented into the medium (Figure 50).
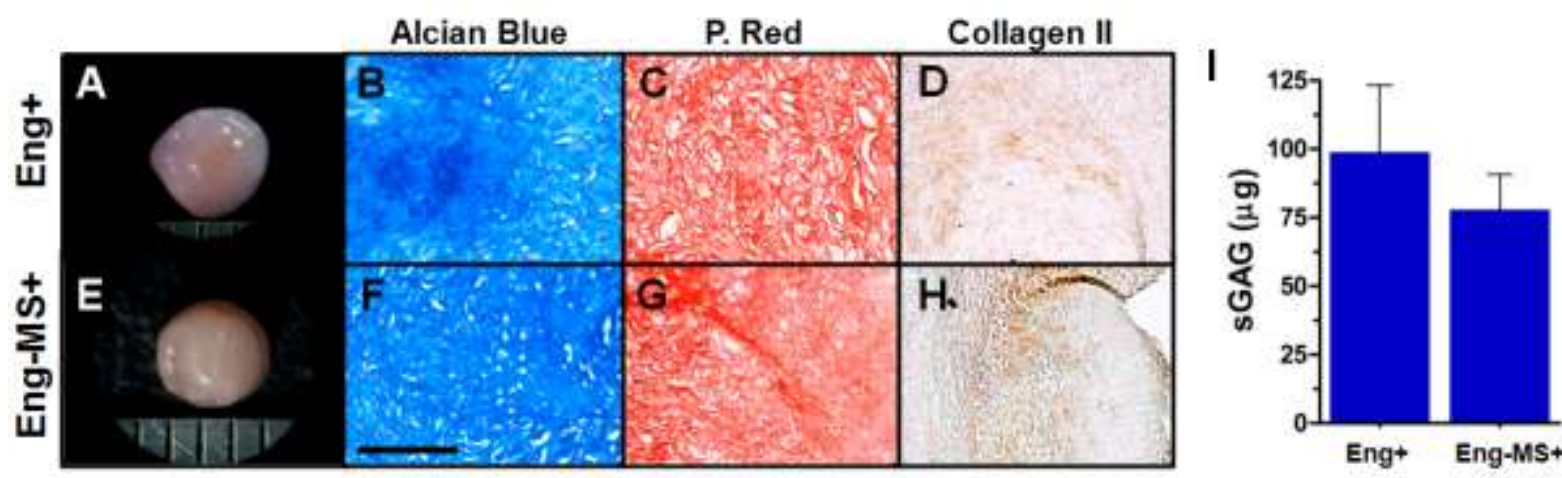

Figure 6. Macroscopic appearance of Eng+ (A) and Eng-MS+ (E) ECM-derived constructs after 28 days in culture (TGF- $\beta 3$ in scaffold). Histological staining for alcian blue, picro-sirius red (P. Red) and type II collagen for Eng+ (B-D) and Eng-MS+ (F-H) groups. All micrographs are for 28 days culture period. sGAG (I) for Eng+ and Eng-MS+ groups with TGF- $\beta 3$ soak-loaded in the scaffold for 28 days in culture $(\mathrm{n}=3)$. Scale bar: $50 \mu \mathrm{m}$.

\section{DISCUSSION}

The purpose of the aforementioned work was to evaluate the potential of devitalized engineered cartilaginous ECM as a biomaterial to fabricate scaffolds for cartilage tissue engineering. We have previously reported that devitalized native porcine articular cartilage ECM-derived scaffolds are chondro-permissive when seeded with human infrapatellar fat pad 
stem cells and cultured in the presence of TGF- $\beta 3{ }^{6 b, 6 c}$. Previous studies have reported that devitalized human engineered cartilage can also be used as a base material to fabricate porous scaffolds to facilitate chondrogenesis of stem cells ${ }^{9 a}$, 9 , although we have shown that such biomaterials can become osteoinductive if the engineered cartilage becomes hypertrophic and begins to mineralize prior to scaffold production ${ }^{9 e}$. The objective of this study was to directly compare the potential of native and engineered cartilage-derived scaffolds to support chondrogenesis of human FPSCs ${ }^{6 b, 6 c}$. The porosity, architecture and mechanical properties of scaffolds produced from engineered and native ECM were similar, although differences in the amount and types of collagen were observed. After seeding with human FPSCs, the native and engineered scaffolds facilitated the formation of a robust cartilage-like tissue. Furthermore, the composition of the devitalized engineered cartilaginous ECM used to fabricate the scaffolds could be modulated by incorporating TGF- $\beta 1$ eluting microspheres into the high density cell sheets used to produce the engineered ECM. Altering the composition of the engineered scaffolds in turn influenced the formation of the cartilaginous tissues that formed within these constructs. These engineered human ECM-derived scaffolds are promising scaffolds for cartilage repair, overcoming limitations associated with the availability and xenogeneic immune reaction to native ECM-derived scaffolds ${ }^{5}$.

Scaffolds derived from ECM engineered in the absence of growth factor releasing MS supported less robust cartilage specific matrix deposition compared to native ECM derived scaffolds. Both scaffolds had a pore size $(74 \pm 28 \mu \mathrm{m}$ for Eng and $75 \pm 31 \mu \mathrm{m}$ Eng-MS scaffolds) and a porosity ( $>90 \%$ ) which has previously been shown to support chondrogenesis ${ }^{6 b, 6 c}$. Furthermore, the initial mechanical properties were similar for both types of scaffolds. However, their composition was different. The native cartilage ECM was richer in type II collagen compared to the engineered tissues. Previous studies have shown that hydrogels and scaffolds functionalized with type II collagen support more robust levels 
of chondrogenesis ${ }^{10 a, 10 c, 21}$. The superior result observed with the native ECM is perhaps not surprising, since the engineered neocartilage was only in culture for 3 weeks prior to devitalization. Nevertheless, care must be taken when comparing type I and II collagen levels in native porcine tissue and engineered human tissue using immunohistochemistry, as it is also possible that the level of antibody reactivity depends on the species, although reactivity with both human and porcine tissue has been reported by the manufacturer. Another difference between native and engineered ECM scaffolds is the level of remnant growth factors. While engineered scaffolds contained higher levels of growth factor, native cartilage is known to contain numerous different growth factors and other chondrogenic cues ${ }^{4 a}$, which may subsequently support chondrogenesis within native ECM-derived scaffolds.

Scaffolds derived from devitalized cartilaginous ECM that was engineered with TGF$\beta 1$ loaded microspheres contained higher levels of remnant TGF- $\beta 3$ than all other scaffolds. This demonstrates that the strategy used to engineer the cartilaginous ECM determines the synthesis of growth factors by MSCs during this period. These higher levels of remnant growth factors could clarify the greater chondrogenesis in Eng-MS scaffolds when compared with Eng groups. Further support for the aforementioned hypothesis is given by the finding that when TGF- $\beta 3$ was directly incorporated in the scaffold prior to cell seeding, the Eng+ and Eng-MS+ ECM-derived scaffolds supported similar levels of cartilage specific matrix deposition, strongly suggesting that local growth factor availability within these scaffolds is critical to ensuring robust chondrogenesis of adult stem cells. When TGF- $\beta 3$ is media supplemented, growth factor transport limitations may occur due to slow diffusion through the construct, as well as preferential TGF- $\beta 3$ uptake by cells in the scaffold periphery ${ }^{9 c,} 22$. Together these results suggest that the specific mode of growth factor presentation will influence the development of cartilaginous constructs within these porous ECM-derived scaffolds. 
Further studies need to be performed with the aim of understanding the role of devitalization/decellularization on the potential of such ECM-derived structures to enhance chondrogenic differentiation and cartilage regeneration, while keeping in mind the immune/inflammatory response. The engineered human ECM-derived scaffolds developed in this study were able to support robust chondrogenesis of FPSCs, especially when loaded with growth factor (TGF- $\beta 3$ ). Engineered tissue maturity also needs to be taken into account, because, for example, the degree of development and composition of the ECM used for scaffold fabrication can dictate chondrogenic growth factor sequestration and availability to the cells ${ }^{3 \mathrm{~d}, 9 \mathrm{~b}}$. It is relevant to plan future studies where scaffold composition is assessed, as the ratio of collagen to GAG will directly influence chondrogenesis of mesenchymal stem cells within such ECM derived biomaterials ${ }^{9 b, 23}$.

\section{CONCLUSION}

This study describes a viable method to use ECM extracted from engineered human cartilage as a material to fabricate chondro-permissive scaffolds for cartilage regeneration. By seeding these scaffolds with infrapatellar fat pad stem cells, it was possible to engineer robust cartilage-like tissues in vitro. The use of such engineered ECM-derived scaffolds could overcome the limitations associated with autogeneic, allogeneic and xenogeneic decellularized tissue grafts. These engineered ECM-derived scaffolds could potentially be used as "off-the-shelf" chondro-permissive scaffolds to support cartilage regeneration.

\section{ACKNOWLEDGMENTS}

Funded by a European Research Council Starter Grant (StemRepair - Project number: 258463), Programme for Research in Third-Level Institutions (PRTLI) - Graduate Research 
Education Programme in Engineering, co-funded by the European Regional Development Fund and the HEA, and the National Institutes of Health (R01AR063194 (EA) and T32AR007505 (ADD)).

\section{REFERENCES}

1. (a) Madeira, C.; Santhagunam, A.; Salgueiro, J. B.; Cabral, J. M., Advanced cell therapies for articular cartilage regeneration. Trends Biotechnol 2015, 33 (1), 35-42; (b) Felson, D. T., Osteoarthritis of the Knee. New England Journal of Medicine 2006, 354 (8), 841-848.

2. (a) Lee, E. J.; Kasper, F. K.; Mikos, A. G., Biomaterials for tissue engineering. Annals of Biomedical Engineering 2014, 42 (2), 323-337; (b) Li, G.; Fu, N.; Xie, J.; Fu, Y.; Deng, S.; Cun, X.; Wei, X.; Peng, Q.; Cai, X.; Lin, Y., Poly(3-hydroxybutyrate-co-4-hydroxybutyrate) based electrospun 3D scaffolds for delivery of autogeneic chondrocytes and adipose-derived stem cells: evaluation of cartilage defects in rabbit. J Biomed Nanotechnol 2015, 11 (1), 105-16; (c) Liao, J.; Qu, Y.; Chu, B.; Zhang, X.; Qian, Z., Biodegradable CSMA/PECA/Graphene porous hybrid scaffold for cartilage tissue engineering. Scientific Reports 2015, 5, 9879.

3. (a) Hutmacher, D. W., Scaffolds in tissue engineering bone and cartilage. Biomaterials 2000, 21 (24), 2529-2543; (b) Even-Ram, S.; Yamada, K. M., Cell migration in 3D matrix. Current Opinion in Cell Biology 2005, 17 (5), 524-532; (c) Pei, M.; He, F.; Kish, V. L., Expansion on extracellular matrix deposited by human bone marrow stromal cells facilitates stem cell proliferation and tissuespecific lineage potential. Tissue engineering. Part A 2011, 17 (23-24), 3067-76; (d) Hynes, R. O., The Extracellular Matrix: Not Just Pretty Fibrils. Science 2009, 326 (5957), 1216-1219.

4. (a) Benders, K. E. M.; Weeren, P. R. v.; Badylak, S. F.; Saris, D. B. F.; Dhert, W. J. A.; Malda, J., Extracellular matrix scaffolds for cartilage and bone regeneration. Trends in Biotechnology 2013, 31 (3), 169-176; (b) Badylak, S. F.; Freytes, D. O.; Gilbert, T. W., Extracellular matrix as a biological scaffold material: Structure and function. Acta biomaterialia 2009, 5 (1), 1-13; (c) Cheng, C. W.; Solorio, L. D.; Alsberg, E., Decellularized tissue and cell-derived extracellular matrices as scaffolds for orthopaedic tissue engineering. Biotechnology advances 2014, 32 (2), 462-484. 
5. Keane, T. J.; Badylak, S. F., The host response to allogeneic and xenogeneic biological scaffold materials. Journal of Tissue Engineering and Regenerative Medicine 2014.

6. (a) Rowland, C. R.; Lennon, D. P.; Caplan, A. I.; Guilak, F., The effects of crosslinking of scaffolds engineered from cartilage ECM on the chondrogenic differentiation of MSCs. Biomaterials 2013, 34 (23), 5802-12; (b) Almeida, H. V.; Cunniffe, G. M.; Vinardell, T.; Buckley, C. T.; O'Brien, F. J.; Kelly, D. J., Coupling Freshly Isolated CD44+ Infrapatellar Fat Pad-Derived Stromal Cells with a TGF- $\beta 3$ Eluting Cartilage ECM-Derived Scaffold as a Single-Stage Strategy for Promoting Chondrogenesis. Advanced Healthcare Materials 2015, 4 (7), 1043-1053; (c) Almeida, H. V.; Liu, Y.; Cunniffe, G. M.; Mulhall, K. J.; Matsiko, A.; Buckley, C. T.; O'Brien, F. J.; Kelly, D. J., Controlled release of transforming growth factor- $\beta 3$ from cartilage-extra-cellular-matrix-derived scaffolds to promote chondrogenesis of human-joint-tissue-derived stem cells. Acta biomaterialia 2014, 10 (10), 4400-9; (d) Yang, Z.; Shi, Y.; Wei, X.; He, J.; Yang, S.; Dickson, G.; Tang, J.; Xiang, J.; Song, C.; Li, G., Fabrication and repair of cartilage defects with a novel acellular cartilage matrix scaffold. Tissue engineering. Part C, Methods 2010, 16 (5), 865-76; (e) Kang, H.; Peng, J.; Lu, S.; Liu, S.; Zhang, L.; Huang, J.; Sui, X.; Zhao, B.; Wang, A.; Xu, W.; Luo, Z.; Guo, Q., In vivo cartilage repair using adipose-derived stem cell-loaded decellularized cartilage ECM scaffolds. J Tissue Eng Regen Med 2014, 8 (6), 442-53; (f) Almeida, H. V.; Eswaramoorthy, R.; Cunniffe, G. M.; Buckley, C. T.; O'Brien, F. J.; Kelly, D. J., Fibrin hydrogels functionalized with cartilage extracellular matrix and incorporating freshly isolated stromal cells as an injectable for cartilage regeneration. Acta biomaterialia 2016, 36, 55-62.

7. (a) Badylak, S. F., Decellularized Allogeneic and Xenogeneic Tissue as a Bioscaffold for Regenerative Medicine: Factors that Influence the Host Response. Annals of Biomedical Engineering 2014, 1-11; (b) Galili, U., Avoiding detrimental human immune response against Mammalian extracellular matrix implants. Tissue engineering. Part B, Reviews 2015, 21 (2), 231-41.

8. Revell, C. M.; Athanasiou, K. A., Success rates and immunologic responses of autogenic, allogenic, and xenogenic treatments to repair articular cartilage defects. Tissue engineering. Part B, Reviews 2009, 15 (1), 1-15. 
9. (a) Lu, H.; Hoshiba, T.; Kawazoe, N.; Chen, G., Autologous extracellular matrix scaffolds for tissue engineering. Biomaterials 2011, 32 (10), 2489-99; (b) Cai, R.; Nakamoto, T.; Kawazoe, N.; Chen, G., Influence of stepwise chondrogenesis-mimicking 3D extracellular matrix on chondrogenic differentiation of mesenchymal stem cells. Biomaterials 2015, 52 (0), 199-207; (c) Solorio, L. D.; Vieregge, E. L.; Dhami, C. D.; Dang, P. N.; Alsberg, E., Engineered cartilage via self-assembled hMSC sheets with incorporated biodegradable gelatin microspheres releasing transforming growth factor-31. Journal of Controlled Release 2012, 158 (2), 224-232; (d) Solorio, L. D.; Dhami, C. D.; Dang, P. N.; Vieregge, E. L.; Alsberg, E., Spatiotemporal regulation of chondrogenic differentiation with controlled delivery of transforming growth factor-beta1 from gelatin microspheres in mesenchymal stem cell aggregates. Stem cells translational medicine 2012, 1 (8), 632-9; (e) Cunniffe, G. M.; Vinardell, T.; Murphy, J. M.; Thompson, E. M.; Matsiko, A.; O’Brien, F. J.; Kelly, D. J., Porous decellularized tissue engineered hypertrophic cartilage as a scaffold for large bone defect healing. Acta biomaterialia 2015, 23, 82-90.

10. (a) Ragetly, G.; Griffon, D. J.; Chung, Y. S., The effect of type II collagen coating of chitosan fibrous scaffolds on mesenchymal stem cell adhesion and chondrogenesis. Acta biomaterialia 2010, 6 (10), 3988-97; (b) Bosnakovski, D.; Mizuno, M.; Kim, G.; Takagi, S.; Okumura, M.; Fujinaga, T., Chondrogenic differentiation of bovine bone marrow mesenchymal stem cells (MSCs) in different hydrogels: influence of collagen type II extracellular matrix on MSC chondrogenesis. Biotechnol Bioeng 2006, 93 (6), 1152-63. (c) Almeida, H. V.; Sathy, B. N.; Dudurych, I.; Buckley, C. T.; O'Brien, F. J.; Kelly, D. J., Anisotropic Shape-Memory Alginate Scaffolds Functionalized with Either Type I or Type II Collagen for Cartilage Tissue Engineering. Tissue Engineering - Part A 2016.

11. Solorio, L. D.; Fu, A. S.; Hernandez-Irizarry, R.; Alsberg, E., Chondrogenic differentiation of human mesenchymal stem cell aggregates via controlled release of TGF-beta1 from incorporated polymer microspheres. Journal of biomedical materials research. Part A 2010, 92 (3), 1139-44.

12. Dikina, A. D.; Strobel, H. A.; Lai, B. P.; Rolle, M. W.; Alsberg, E., Engineered cartilaginous tubes for tracheal tissue replacement via self-assembly and fusion of human mesenchymal stem cell constructs. Biomaterials 2015, 52, 452-62. 
13. (a) Haugh, M. G.; Murphy, C. M.; McKiernan, R. C.; Altenbuchner, C.; O'Brien, F. J., Crosslinking and mechanical properties significantly influence cell attachment, proliferation, and migration within collagen glycosaminoglycan scaffolds. Tissue Engineering - Part A 2011, 17 (9-10), 1201-1208; (b) Olde Damink, L. H. H.; Dijkstra, P. J.; Van Luyn, M. J. A.; Van Wachem, P. B.; Nieuwenhuis, P.; Feijen, J., In vitro degradation of dermal sheep collagen cross-linked using a watersoluble carbodiimide. Biomaterials 1996, 17 (7), 679-684.

14. Guillaume, O.; Daly, A.; Lennon, K.; Gansau, J.; Buckley, S. F.; Buckley, C. T., Shapememory porous alginate scaffolds for regeneration of the annulus fibrosus: effect of TGF-beta3 supplementation and oxygen culture conditions. Acta biomaterialia 2014, 10 (5), 1985-95.

15. Buckley, C. T.; Vinardell, T.; Thorpe, S. D.; Haugh, M. G.; Jones, E.; McGonagle, D.; Kelly, D. J., Functional properties of cartilaginous tissues engineered from infrapatellar fat pad-derived mesenchymal stem cells. Journal of Biomechanics 2010, 43 (5), 920-926.

16. (a) Ignat'eva, N. Y.; Danilov, N. A.; Averkiev, S. V.; Obrezkova, M. V.; Lunin, V. V.; Sobol, E. N., Determination of hydroxyproline in tissues and the evaluation of the collagen content of the tissues. Journal of Analytical Chemistry 2007, 62 (1), 51-57; (b) Buckley, C. T.; Kelly, D. J., Expansion in the presence of FGF-2 enhances the functional development of cartilaginous tissues engineered using infrapatellar fat pad derived MSCs. Journal of the mechanical behavior of biomedical materials 2012, 11, 102-111.

17. Gannon, A. R.; Nagel, T.; Kelly, D. J., The role of the superficial region in determining the dynamic properties of articular cartilage. Osteoarthritis and cartilage / OARS, Osteoarthritis Research Society 2012, 20 (11), 1417-25.

18. Sheehy, E. J.; Mesallati, T.; Vinardell, T.; Kelly, D. J., Engineering cartilage or endochondral bone: A comparison of different naturally derived hydrogels. Acta biomaterialia 2015, 13 (0), 245253.

19. Albro, M. B.; Nims, R. J.; Cigan, A. D.; Yeroushalmi, K. J.; Alliston, T.; Hung, C. T.; Ateshian, G. A., Accumulation of exogenous activated TGF-beta in the superficial zone of articular cartilage. Biophysical journal 2013, 104 (8), 1794-804. 
20. Vonwil, D.; Wendt, D.; Ströbel, S.; Wallny, H. J.; Gygax, D.; Heberer, M.; Martin, I., Assessment of the stability of TGF $\beta 3$ bioactivity for potential bioreactor applications. Biochemical Engineering Journal 2008, 39 (3), 586-589.

21. (a) Rutgers, M.; Saris, D. B.; Vonk, L. A.; van Rijen, M. H.; Akrum, V.; Langeveld, D.; van Boxtel, A.; Dhert, W. J.; Creemers, L. B., Effect of collagen type I or type II on chondrogenesis by cultured human articular chondrocytes. Tissue engineering. Part A 2013, 19 (1-2), 59-65; (b) Ragetly, G. R.; Griffon, D. J.; Lee, H. B.; Chung, Y. S., Effect of collagen II coating on mesenchymal stem cell adhesion on chitosan and on reacetylated chitosan fibrous scaffolds. Journal of materials science. Materials in medicine 2010, 21 (8), 2479-90.

22. (a) Johnstone, B.; Hering, T. M.; Caplan, A. I.; Goldberg, V. M.; Yoo, J. U., In vitro chondrogenesis of bone marrow-derived mesenchymal progenitor cells. Experimental Cell Research 1998, 238 (1), 265-272; (b) Albro, M. B.; Nims, R. J.; Durney, K. M.; Cigan, A. D.; Shim, J. J.; Vunjak-Novakovic, G.; Hung, C. T.; Ateshian, G. A., Heterogeneous engineered cartilage growth results from gradients of media-supplemented active TGF-beta and is ameliorated by the alternative supplementation of latent TGF-beta. Biomaterials 2016, 77, 173-85.

23. (a) Derfoul, A.; Miyoshi, A. D.; Freeman, D. E.; Tuan, R. S., Glucosamine promotes chondrogenic phenotype in both chondrocytes and mesenchymal stem cells and inhibits MMP-13 expression and matrix degradation. Osteoarthritis and Cartilage 2007, 15 (6), 646-655; (b) Varghese, S.; Theprungsirikul, P.; Sahani, S.; Hwang, N.; Yarema, K. J.; Elisseeff, J. H., Glucosamine modulates chondrocyte proliferation, matrix synthesis, and gene expression. Osteoarthritis and Cartilage 2007, 15 (1), 59-68. 
"For table of contents only"

Title: "Porous Scaffolds Derived from Devitalized Tissue Engineered Cartilaginous Matrix Support Chondrogenesis of Adult Stem Cells".

Authors: Henrique V. Almeida, Anna D. Dikina, Kevin J. Mulhall, Fergal J. O’Brien, Eben Alsberg, Daniel J. Kelly.

Human

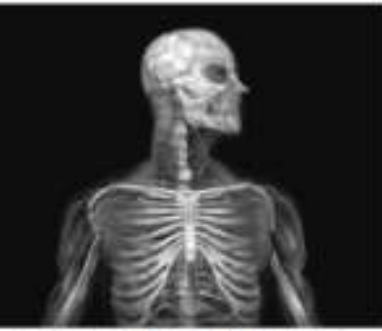

Human Bone Marrow Stem Cells (MSCs)

In vitro Cartilage Engineering

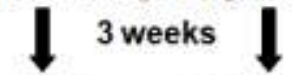

MSC MSC+MS

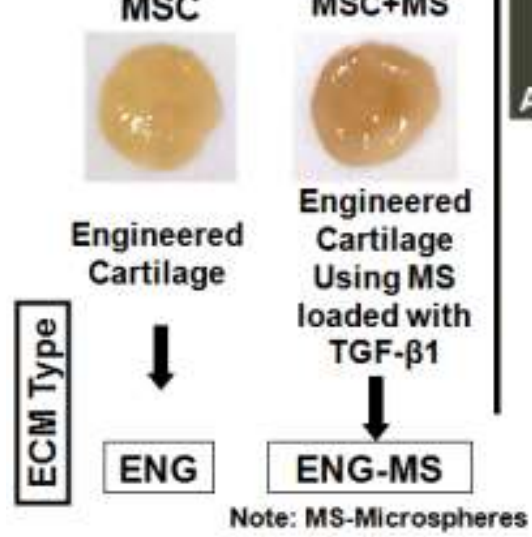

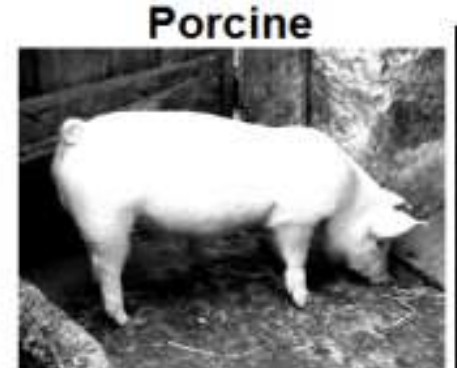

Native Cartilage ECM

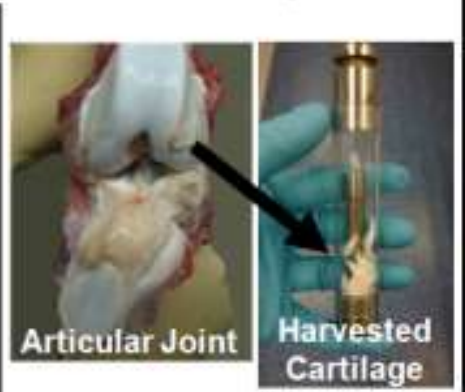

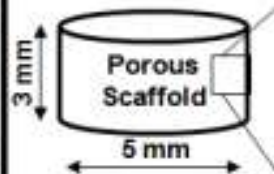

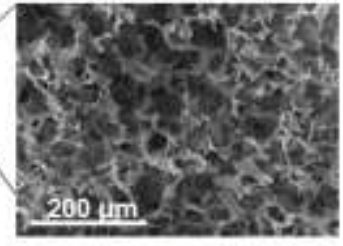

Cartilage ECM Scaffold
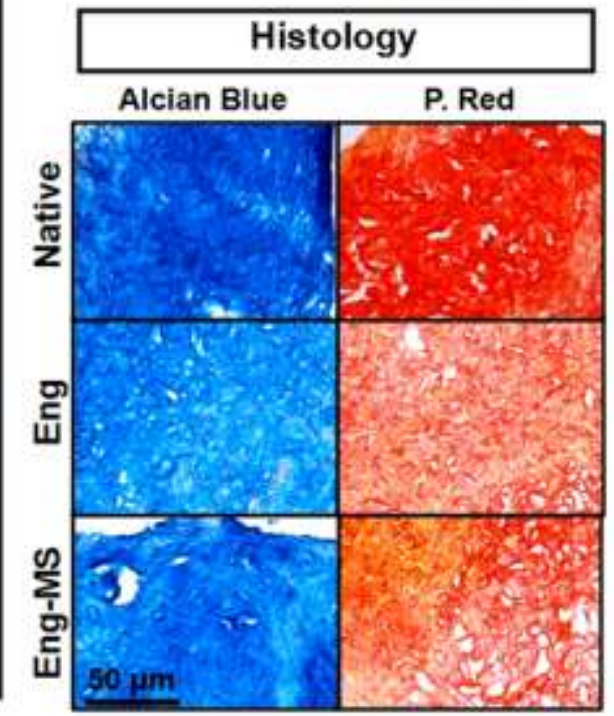